\section{A Large-Scale Deforestation Experiment: Effects of Patch Area and Isolation on Amazon Birds}

Gonçalo Ferraz, ${ }^{1 *}$ ]ames D. Nichols, ${ }^{2}$ James E. Hines, ${ }^{2}$ Philip C. Stouffer, ${ }^{1,3}$ Richard O. Bierregaard Jr., ${ }^{1,4}$ Thomas E. Lovejoy ${ }^{1,5}$

As compared with extensive contiguous areas, small isolated habitat patches lack many species. Some species disappear after isolation; others are rarely found in any small patch, regardless of isolation. We used a 13-year data set of bird captures from a large landscape-manipulation experiment in a Brazilian Amazon forest to model the extinction-colonization dynamics of 55 species and tested basic predictions of island biogeography and metapopulation theory. From our models, we derived two metrics of species vulnerability to changes in isolation and patch area. We found a strong effect of area and a variable effect of isolation on the predicted patch occupancy by birds.

$\mathrm{T}$ ropical forests are among the world's most threatened ecosystems, which are under severe pressure by human activities, primarily deforestation (1). Deforestation reduces forest area and frequently isolates remaining forest patches. Island biogeography (2) and metapopulation (3) theories predict the effects of reduced area and increased isolation on rates of species extinction and colonization and thus on species occurrence. However, strong tests of these predictions are scarce. Most published inferences about the effects of area and isolation are based on patterns of species occurrence, rather than directly on the dynamic rate parameters (such as extinction and colonization) that produce these patterns. Multiple plausible hypotheses can be developed to explain any such pattern, and pattern-based analyses thus produce weak inferences (4). The few studies that have focused on patch-occupancy dynamics follow observational, rather than experimental, designs at small spatial and temporal scales, and they fail to deal adequately with detection probabilities: the fact that species may be present at a site yet go undetected.

The Biological Dynamics of Forest Fragments Project (BDFFP) was initiated to test the effects of forest destruction at the patch level in a tropical ecosystem (5). The study, located in the central Brazilian Amazon, involved a reduction in forest area, which was predicted to increase the probabilities of local extinction of species, and isolation of remnant forest patches, which was predicted to reduce species colonization probabilities and increase extinction $(2,3,6)$. The combined effects of both factors are pre-

${ }^{1}$ Biological Dynamics of Forest Fragments Project, Instituto Nacional de Pesquisas da Amazônia, 69011 Manaus AM, Brazil. ${ }^{2}$ Patuxent Wildlife Research Center, Laurel, MD 20708, USA. ${ }^{3}$ School of Renewable Natural Resources, Louisiana State University (LSU) and LSU AgCenter, Baton Rouge, LA 70803, USA. ${ }^{4}$ Department of Biology, University of North Carolina, Charlotte, NC 28223, USA. ${ }^{5}$ The H. John Heinz III Center for Science Economics and the Environment, Washington, DC 20004, USA.

*To whom correspondence should be addressed. E-mail: gferraz@inpa.gov.br dicted to reduce patch occupancy $(2,3)$. We tested these predictions using data from the diverse bird community while separating inferences about area and isolation, in contrast to more usual approaches that confound these two effects as "fragmentation" (7). The data are of an experimental nature on relevant geographical and temporal scales, and our analysis treats detection probabilities explicitly.

A mist-netting program monitored understory birds in 23 primary-forest patches for 13 years (5). All patches were initially in continuous forest, but 11 of them were subsequently isolated by ranchland (two of which are depicted in Fig. 1). Patches were set in size classes of 1 , $10,100,500$, and 600 ha, with the largest isolated patch at 100 ha (table S1). The forest was cleared after monitoring began, permitting inference about the processes of extinction and colonization in isolated and continuous forest patches of different sizes (8).

We used patch-occupancy models (9) to test a priori hypotheses about the influence of patch area and isolation on occurrence dynamics of 55 well-sampled bird species. The models contain four kinds of parameters: initial occupancy $\left(\psi_{1}\right)$, local extinction probability $(\varepsilon)$, local probability of colonization $(\gamma)$, and probability of detection given presence $(p)$. We limited our model set to plausible a priori

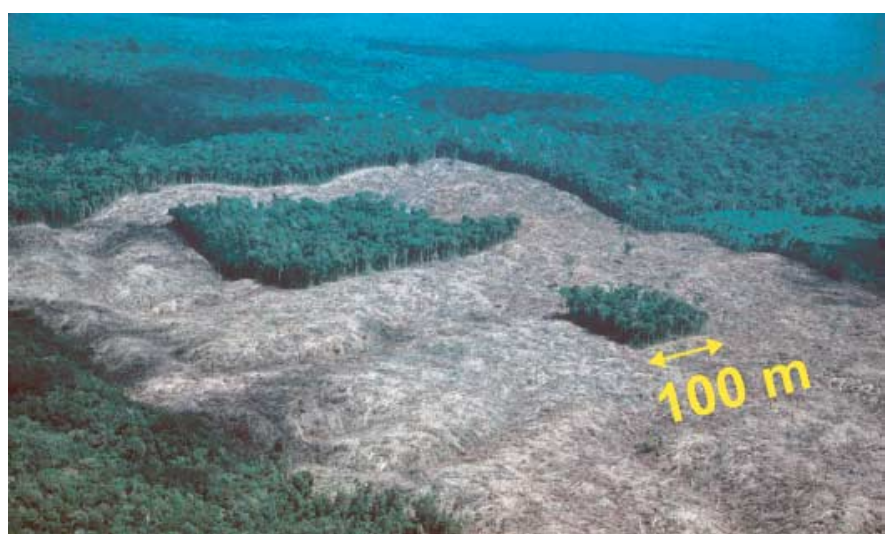

hypotheses about the processes of detection, colonization, and local extinction, rather than conducting exploratory analyses with a larger model set including various combinations of potential covariates (table S2). $\psi_{1}$ is a free parameter and is never related to a covariate. $p$ is a function of mist-netting effort and takes different values for different species. We predicted that $\varepsilon$ would be related to patch area and isolation in one of three ways: a multiplicative function of patch size and isolation (full interaction model), an additive function of the same two variables, and a function of patch size alone. $\gamma$ should be related to isolation, perhaps as modified by regrowth of matrix habitat because some ranchland was abandoned. Colonization is modeled as a function of isolation, regrowth, and the "year 1 effect"-increased colonization by displaced birds immediately after deforestation - taken in five additive combinations. Isolation is a binary variable. We thus fitted $3 \times 5=15$ different models to each species using a maximum-likelihood approach in the program PRESENCE (10). Models were ranked by Akaike's information criterion (AIC) and AIC weight $w_{j}$ for model $j$ (11). We predicted that patch size, independently of isolation, should have a negative effect on $\varepsilon(2,3)$. Isolation, independently of size, should have a positive effect on local extinction via the reduction of the "rescue effect" ( 6 ).

We also asked three expert neotropical ornithologists to classify the study species into two categories of dispersal ability (low and high). Isolation, through its effects on colonization and local extinction, should be more important for models of poor dispersers than for models of good dispersers. We expected forest regrowth to be more important for poor dispersers than for good dispersers. Among the variables that affect $\varepsilon$ and $\gamma$, we predicted that dispersal ability would be the most relevant in the species' responses to deforestation. Other variables may also be relevant, but their predicted effects are typically based on their relationship to dispersal ability. Thus, rather than testing the relevance of various species classification schemes, we focus here on our a priori prediction about the relevance of dispersal ability.

Fig. 1. Two isolated fragments at Fazenda Dimona, Brazil. This aerial photograph of a 10-ha and a 1-ha fragment was taken shortly after isolation. Gaps between the fragment edge and the continuous forest are less than $1 \mathrm{~km}$ wide. 
Our analysis follows three steps, all of which are based on fitting models for each species. First, we ask what covariates appear in the topranking models of each species. Second, we focus on the single model that fits best across species and examine the signs and magnitudes of covariate effects (slope parameters). Finally, we select one best-fitting model per species and draw inferences based on the estimated local extinction and colonization parameters.

Patch isolation appears as a covariate of colonization and/or local extinction in highranking models $\left(w_{j}>0.2\right)$ of nearly all, but not all, species (Table 1 and table S3). Patch size, regardless of isolation, seems sufficient to explain the observations on three exception species: Geotrygon montana, Dendrocolaptes certhia, and Hypocnemis cantator. Fifteen species have high-ranking models with an effect of isolation only on local extinction, and five species have high-ranking models with that effect only on colonization. Regrowth enters high-ranking models in two-fifths of the species. Contrary to our expectation, we cannot reject the null hypothesis that regrowth enters highranking models of high- and low-dispersal species in the same proportions (one-tailed $z$ test, $P=0.33$ ). Likewise, there is no evidence to sustain the prediction that isolation would appear more frequently in high-ranking models of low-dispersal species (one-tailed $z$ test, $P=0.97$ ). Inferences are qualitatively the same if highranking models are redefined as having $w_{j}>$ 0.1 or $w_{j}>0.25$.

Comparison of estimated slope parameters for area and isolation across species is facilitated by use of a single model, so we focus on model 6 (table S2 and Fig. 2), which has the highest average $w_{j}$ across species. Model 6 hypothesizes fixed colonization and an additive effect of size and isolation on local extinction. There is a negative effect of patch size on $\varepsilon$ : For all species, larger plots have lower $\varepsilon$ values. Isolation shows more variable results, with 36 of 55 species showing a positive slope. As predicted, the effect of isolation on local extinction is positive more often than negative (one-tailed $z$ test, $P<$ 0.01 ), but for roughly one-third of the species, slope estimates are very close to zero (or even negative). We found no evidence to suggest that

Table 1. Contribution of isolation and regrowth covariates to high-ranking models of 50 species, grouped by dispersal ability. Isolation is a binary variable (" 1 " for isolated patches and " 0 " for otherwise). Regrowth counts the number of years since isolation. Five species had no models with $w_{j} \geq 0.2$.

Number of species

\begin{tabular}{|c|c|c|c|c|}
\hline \multirow{2}{*}{$\begin{array}{l}\text { Does the isolation or regrowth } \\
\text { covariate enter any part of a model } \\
\text { with } w_{j} \geq 0.2 \text { ? }\end{array}$} & \multicolumn{2}{|c|}{ Isolation } & \multicolumn{2}{|c|}{ Regrowth } \\
\hline & Yes & No & Yes & No \\
\hline \multicolumn{5}{|l|}{ Dispersal ability of species } \\
\hline Low & 25 & 3 & 11 & 17 \\
\hline High & 22 & 0 & 9 & 13 \\
\hline
\end{tabular}

largest to the smallest continuous-forest (cf) patch:

$$
A=1-\frac{\psi_{\mathrm{cf}_{1}}^{*}}{\psi_{\mathrm{cf}_{100}}^{*}}
$$

with the numerical subscript denoting patch size (in hectares). The uncommon blackthroated antshrike (Frederickena viridis), a forest-interior antbird with narrow habitat requirements, exemplifies a species that is highly sensitive to area (Fig. 3, D to F). Poor colonizers that rarely cross open areas, or species that do not survive well in isolation, should have relatively low $\psi_{\mathrm{i}_{\mathrm{s}}^{*}}^{*}$ values in isolated patches. Thus, we measure vulnerability to isolation $I$ (Fig. 2C) as the relative reduction in $\psi_{\mathrm{i}_{\mathrm{s}}}^{*}$ from 1-ha continuous-forest to 1-ha isolated (isol) patches:

$$
I=1-\frac{\psi_{\text {isol }_{1}}^{*}}{\psi_{\mathrm{cf}_{1}}^{*}}
$$

The white-chinned woodcreeper (D. merula), a bird that forages by following swarms of army ants, is highly vulnerable to isolation but not sensitive to area (Fig. 3, A to C).

We use approximate $95 \%$ confidence intervals (Fig. 4) to assess whether each metric is significantly different from zero. In agreement with results based on slope parameters and $w_{j}$, a substantial proportion (29/54) of species is not significantly vulnerable to isolation. There is a
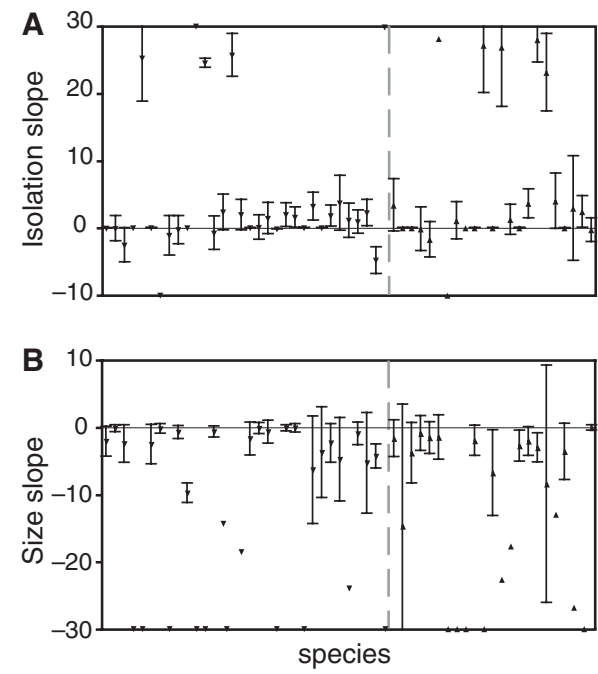

Fig. 2. Slope parameter estimates for the effect of isolation (A) and patch size (B) on local extinction, as according to model 6. Low- and high-dispersal species (table S3) appear on the left and right sides of the dashed line, respectively. Error bars indicate approximate 95\% confidence intervals, assuming normally distributed parameter estimates. Points without confidence intervals on the upper or lower edges of the plot indicate point estimates beyond the limits of the $y$ axis. Confidence intervals could not be estimated for all points. 
Fig. 3. Example of patch-occupancy parameters estimated by the bestfitting models for two species. Solid and open circles show estimates for continuous forest and isolated patches, respectively; error bars and lines without symbols show 95\% confidence intervals. $\psi_{i_{s}}^{*}$ (C and $\mathbf{F}$ ) is a function of $\varepsilon$ ( $\mathbf{A}$ and $\mathbf{D}$ ) and $\gamma$ (B and $\mathbf{E})$. D. merula $[(\mathrm{A})$ to (C)] shows a marked effect of isolation both on local extinction and on colonization and a stronger effect on $\psi_{i_{\text {s }}}^{*}$. Estimates for $F$. viridis [(D) to $(F)]$ vary mostly with patch area. Arrows in (C) illustrate two metrics reflecting use of space: $A$ and $I$.

Fig. 4. Values of $A$ and $/$ for 54 species grouped by uncertainty over the two metrics (table S4). Open and solid circles indicate that the approximate $95 \%$ confidence intervals of both metrics or of neither metric overlap zero, respectively. Upward and rightward pointing triangles indicate that only the confidence interval of $A$ or I does not overlap zero, respectively. Symbols with arrows indicate point estimates outside the plotted range of the $x$ axis.

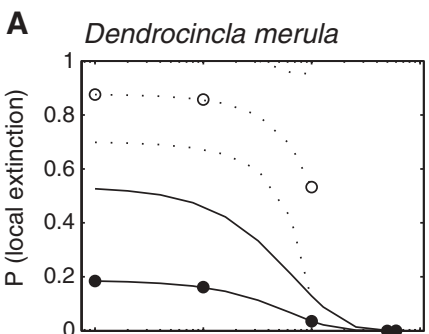

B
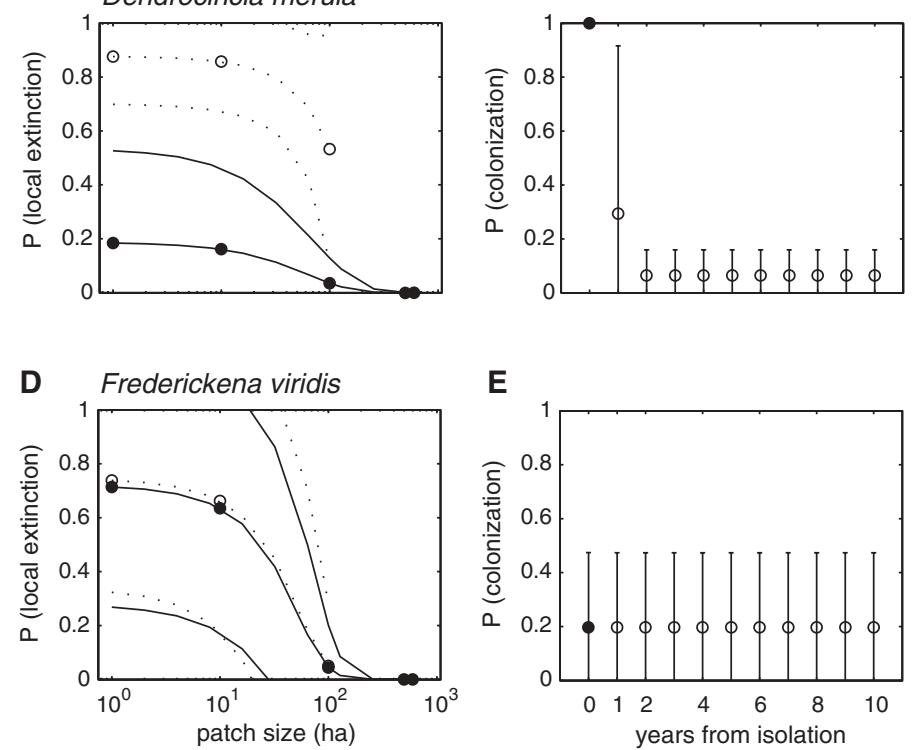

E

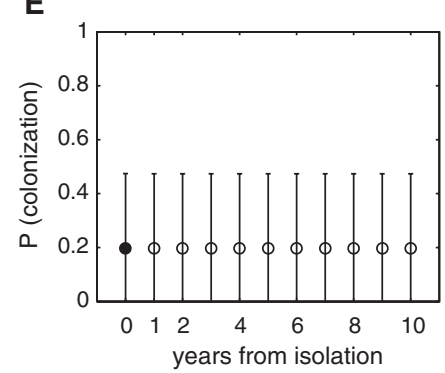

C

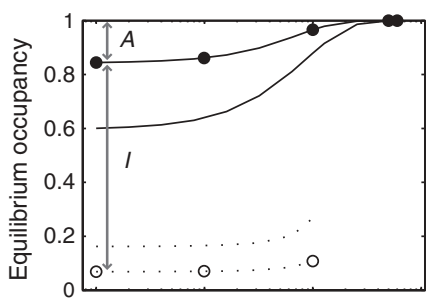

$\mathbf{F}$

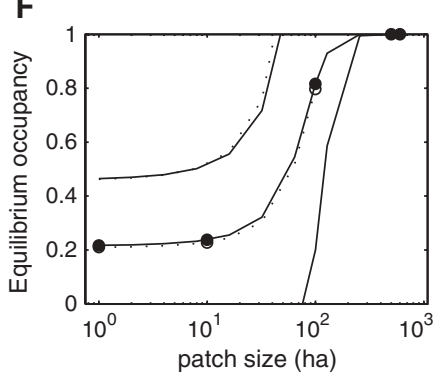

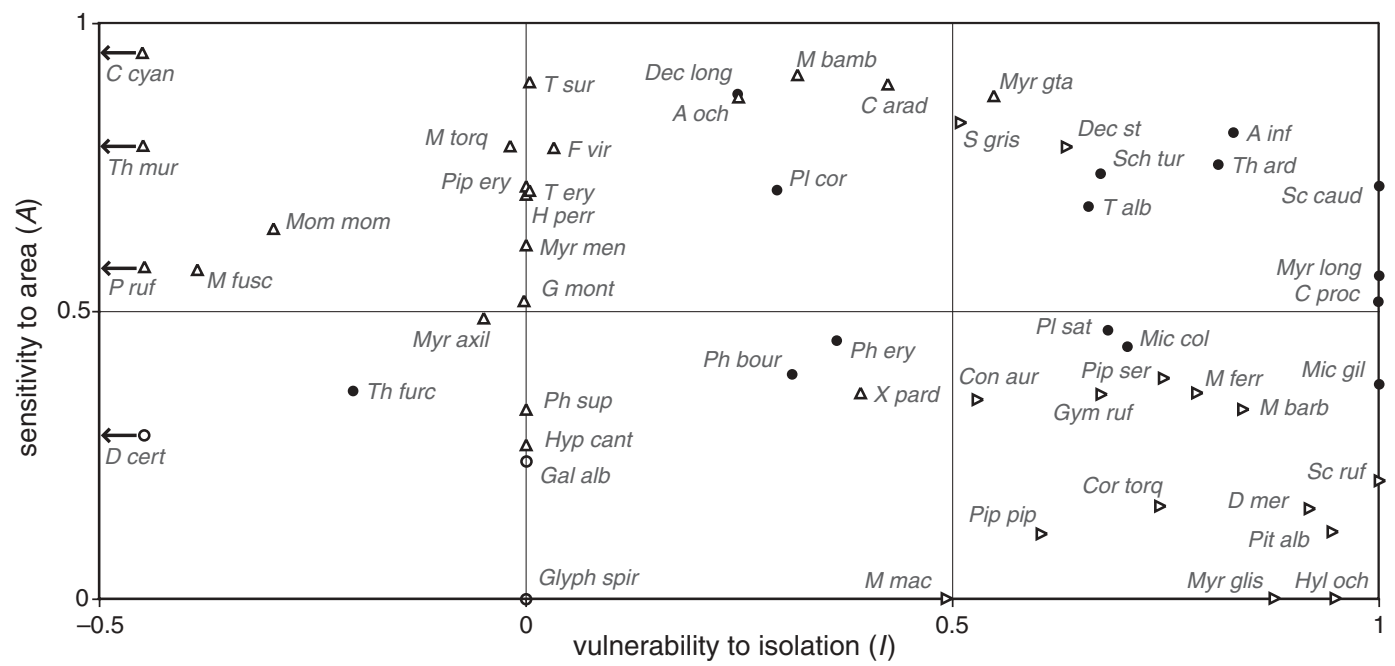

higher proportion $(36 / 54)$ of species with area sensitivity that is significantly different from zero, with two-thirds of these species not being significantly vulnerable to isolation. This result suggests that many species fail to occur in small isolated patches, not because they succumb to the effects of isolation in reducing any rescue effect but because they rarely occupy any small patch (even in continuous forest) just because of their pattern of space use. Management aimed at curbing the effects of isolation (e.g., corridors and edge protection) will have reduced effectiveness for those species that are highly sensitive to area.

Our finding that local extinctions are always more probable in small than in large patches is predicted by demographic theory and is evident from the slope parameters of model 6 . The role of isolation is less obvious: A predominantly positive but variable effect of isolation on local extinction under model 6 must be qualified by the many low and uncertain estimates of $I$. Our knowledge of the dispersal abilities of species in undisturbed conditions does not explain the variation in the signs of the slope parameters or in the contributions of the isolation and regrowth covariates to highranking models. Either we do not know enough about dispersal abilities - a notion that is widely supported by neotropical ornithologists - or those abilities change in disturbed landscapes to the extent that we cannot use them to predict occupancy parameters under disturbance.

Deforestation results in forest-area reduction and isolation of remaining forest patches, and both factors affect occupancy dynamics. This study shows the generality of these effects by showing that they apply to tropical forest landscapes in addition to the temperate landscapes from which most previous inferences have come. The interspecific variation in the effects of isolation on the studied tropical bird community is reminiscent of similar variation reported for avian communities in temperate forests of North America (15). Both the North American study and our more realistic modeling approach report interspecific variation, but they differ in that our finding demonstrated a relatively stronger effect of area than of isolation. We qualify this finding by noting that our data come from a landscape that is dominated by forest. The distance between isolated patches and continuous forest ranges from 150 to $900 \mathrm{~m}$ (16). Further forest destruction is expected to result in additional species loss and larger effects of isolation $(7,17)$.

References and Notes

1. W. F. Laurance, C. A. Peres, Eds. Emerging Threats to Tropical Forests (University of Chicago Press, Chicago, 2006).

2. R. H. MacArthur, E. O. Wilson, The Theory of Island Biogeography (Princeton Univ. Press, Princeton, 1967).

3. I. Hanski, Metapopulation Ecology (Oxford Univ. Press, Oxford, 1999).

4. D. I. MacKenzie et al., Occupancy Estimation and Modeling: Inferring Patterns and Dynamics of Species Occurrence (Academic Press, Amsterdam, 2006).

5. R. O. Bierregaard Jr., C. Gascon, T. E. Lovejoy, R. C. G. Mesquita, Eds. Lessons from Amazonia: The Ecology and Conservation of a Fragmented Forest (Yale Univ. Press, New Haven, 2001). 
6. J. H. Brown, A. Kodric-Brown, Ecology 58, 445 (1977).

7. L. Fahrig, Annu. Rev. Ecol. Evol. Syst. 34, 487 (2003).

8. Materials and methods are available as supporting material on Science Online.

9. D. I. MacKenzie, J. D. Nichols, ]. E. Hines, M. G. Knutson, A. B. Franklin, Ecology 84, 2200 (2003).

10. J. E. Hines, PRESENCE version 2.0 (computer program), U.S. Geological Survey, Patuxent Wildlife Research Center, Laurel, MD (2004).

11. K. P. Burnham, D. R. Anderson, Model Selection and Multimodel Inference: A Practical Information-Theoretic Approach (Springer, New York, 2002).

12. P. C. Stouffer, R. O. Bierregaard Jr., Ecology 76, 2429 (1995)
13. K. S. Van Houtan, S. L. Pimm, R. O. Bierregaard Jr., T. E. Lovejoy, P. C. Stouffer, Evol. Ecol. Res. 8, 129 (2006).

14. M. Cohn-Haft, A. Whittaker, P. C. Stouffer, Ornithol. Monogr. 48, 205 (1997)

15. C. S. Robbins, D. K. Dawson, B. A. Dowell, Wildl. Monogr. 103, 1 (1989).

16. C. Gascon, R. O. Bierregaard Jr., in (5), pp. 31-45.

17. M. K. Trzcinski, L. Fahrig, G. Merriam, Ecol. Appl. 9, 586 (1999).

18. We thank A. Whittaker, ].-M. Thiollay, and S. Borges for help with classification of dispersal abilities. L. Naka and M. Cohn-Haft provided key natural history information.
G.F. was supported by BDFFP funds and a Conselho Nacional de Desenvolvimento Científico e Tecnológico (Brazilian Government) grant. This is BDFFP technical series contribution number 473 .

Supporting Online Material

www.sciencemag.org/cgi/content/ful//315/5809/238/DC1

Materials and Methods

Fig. S1

Tables S1 to S4

References

27 July 2006; accepted 28 November 2006 $10.1126 /$ science. 1133097

\section{Distinct Populations of Primary and Secondary Effectors During RNAi in C. elegans}

Julia Pak and Andrew Fire*

RNA interference (RNAi) is a phylogenetically widespread gene-silencing process triggered by double-stranded RNA. In plants and Caenorhabditis elegans, two distinct populations of small RNAs have been proposed to participate in RNAi: "Primary siRNAs" (derived from DICER nucleasemediated cleavage of the original trigger) and "secondary siRNAs" [additional small RNAs whose synthesis requires an RNA-directed RNA polymerase (RdRP)]. Analyzing small RNAs associated with ongoing RNAi in C. elegans, we found that secondary siRNAs constitute the vast majority. The bulk of secondary siRNAs exhibited structure and sequence indicative of a biosynthetic mode whereby each molecule derives from an independent de novo initiation by RdRP. Analysis of endogenous small RNAs indicated that a fraction derive from a biosynthetic mechanism that is similar to that of secondary siRNAs formed during RNAi, suggesting that small antisense transcripts derived from cellular messenger RNAs by RdRP activity may have key roles in cellular regulation.

$\mathrm{D}$ ouble-stranded RNA (dsRNA)-triggered gene silencing in eukaryotes appears universally to involve 21 - to 25 -nucleotide (nt) siRNA effectors. In Drosophila and mammals, siRNAs derive primarily from processing of longer duplexes by DICER nuclease, forming 21to 25-nt duplexes possessing 5'-monophosphates, 3'-hydroxyl groups, and 2-nt 3' overhangs $(1,2)$. Along with this "primary" siRNA response, amplification of the RNA trigger population has been proposed to contribute to potency and persistence of gene silencing in several systems $(3,4)$. Amplification mechanisms are accompanied in some cases by "transitive RNAi" phenomena in which dsRNA matching one mRNA region can silence targets bearing homology to other parts of the mRNA (5-9). Unlike the situation in plants where "spreading" of the effector population occurs bidirectionally relative to the target mRNA $(6,8)$, transitive RNAi in Caenorhabditis elegans exhibits a strong bias toward sequences upstream of trigger homology $(7,9)$. Transitive RNAi requires function of a putative RdRP (RRF-1 in C. elegans soma, SDE1/SGS2 in Arabidopsis thaliana), suggesting several conceivable means for secondary siRNA production

Departments of Pathology and Genetics, Stanford University School of Medicine, Stanford, CA 94305-5324, USA.

*To whom correspondence should be addressed. E-mail: afire@stanford.edu
$(6,7)$. One possibility is that antisense primary siRNAs could act as primers in the RdRPmediated synthesis of new dsRNAs on an mRNA template. Alternatively, primary siRNAs may merely guide the RdRP to a target, allowing unprimed synthesis $(10,11)$ either at the cleaved end of the targeted transcript, at a location close to the trigger-target complex, or at a structure such as a free end that might be revealed as aberrant through consequences of the initial RNA-induced silencing complex (RISC)::target interaction.

To better understand signal amplification in C. elegans, we characterized small RNAs from animals undergoing RNAi against an abundantly expressed endogenous gene, sel-1 (12). After reverse transcription, 245,420 18- to 25-nt RNAs were sequenced by means of single-molecule pyrosequencing (13). Among these sequences, 534 exhibited either a perfect match (428 instances) or single mismatches (106 instances) to sel-1 mRNA (Fig. 1, A and B). A similar analysis of $\sim 850,000$ clones from animals not exposed to dsRNA yielded just one sel-1 small RNA (14). Most sel-1 small RNAs induced during interference (483) had an antisense orientation, consistent with previous hybridization-based analyses (7). Of the 51 sense strand clones, 22 showed complementarity to at least one antisense clone.

We observed an incomplete bias in siRNA positions relative to the trigger; of 138 antisense siRNAs outside the original trigger, $110(80 \%)$ occurred on the $5^{\prime}$ side. This bias could certainly account for preferential detection of upstream secondary responses in functional and biochemical assays $(7,9)$. Twenty-eight observed instances of small antisense RNAs completely downstream of the trigger homology were of particular interest, as these would not have been expected if the sole mode of amplification involved extension by RdRP of existing siRNA triggers that hybridize to the target transcript.

Exon-exon junctions offer a unique opportunity to unequivocally distinguish de novo synthesis of antisense nucleic acids from an mRNA template $(15,16)$. We found 50 sel-1 small antisense RNA sequences that span exon/exon junctions. Of these, 43 fall within the trigger [ 458 base pairs (bp) of sel-1 cDNA sequence] and thus could have derived directly from triggering dsRNA. Six antisense exon-exon junction sequences upstream of the trigger were recovered (four matching perfectly and two with single mismatches). These imply de novo copying of the mature mRNA template (16).

The apparent scarcity of sel-1 siRNAs suggested that the procedure for cloning small RNAs (including ligation of linkers to $3^{\prime}$ and $5^{\prime}$ ends) might underrepresent the siRNA population (12). To analyze small RNA termini in detail, we used a number of structure-specific treatments. Treatment of RNA with periodate followed by $\beta$ elimination results in a shift on a denaturing acrylamide gel, indicating at least one unmodified (cis-diol) $3^{\prime}$ terminus (17). Ribonuclease $\mathrm{T}$ (RNaseT) requires a 3'-hydroxyl to degrade single-stranded RNA. Finally, Terminator exonuclease preferentially degrades substrates with a single 5'-phosphate. Although sel-1 siRNAs are susceptible to both $\beta$ elimination and RNaseT reactions, they are resistant to Terminator (Fig. 2A). Control synthetic 25-nt sel-1 RNA oligonucleotides $\mathrm{s}$ with 5 '-monophosphate and $3^{\prime}-\mathrm{OH}$ were sensitive to all three treatments. We surmised that sel-1 siRNAs are blocked at their 5 ' ends.

We next asked if we could design a cloning protocol that would not be biased by the structure at the $5^{\prime}$ end on an siRNA. The resulting protocol (fig. S3) avoids both (i) the requirement for ligation of the $5^{\prime}$ end of the RNA and (ii) the possibility that modified $5^{\prime}$ ends on small RNAs could affect enzymatic treatments of the paired cDNA strand. We detected 127 sel-1 antisense sequences and zero sense sequences from 1612 